\title{
Genomic, regulatory and epigenetic mechanisms underlying duplicated gene evolution in the natural allotetraploid Oryza minuta
}

Yi Suil ${ }^{1,2+}$, Bo Li ${ }^{1+}$, Jinfeng Shi ${ }^{1}$ and Mingsheng Chen ${ }^{1 *}$

\begin{abstract}
Background: Polyploid species contribute to Oryza diversity. However, the mechanisms underlying gene and genome evolution in Oryza polyploids remain largely unknown. The allotetraploid Oryza minuta, which is estimated to have formed less than one million years ago, along with its putative diploid progenitors (O. punctata and $O$. officinalis), are quite suitable for the study of polyploid genome evolution using a comparative genomics approach.

Results: Here, we performed a comparative study of a large genomic region surrounding the Shattering 4 locus in O. minuta, as well as in O. punctata and O. officinalis. Duplicated genomes in O. minuta have maintained the diploid genome organization, except for several structural variations mediated by transposon movement. Tandem duplicated gene clusters are prevalent in the Sh4 region, and segmental duplication followed by random deletion is illustrated to explain the gene gain-and-loss process. Both copies of most duplicated genes still persist in O. minuta. Molecular evolution analysis suggested that these duplicated genes are equally evolved and mostly manipulated by purifying selection. However, CDNA-SSCP analysis revealed that the expression patterns were dramatically altered between duplicated genes: nine of 29 duplicated genes exhibited expression divergence in O. minuta. We further detected one gene silencing event that was attributed to gene structural variation, but most gene silencing could not be related to sequence changes. We identified one case in which DNA methylation differences within promoter regions that were associated with the insertion of one $h A T$ element were probably responsible for gene silencing, suggesting a potential epigenetic gene silencing pathway triggered by TE movement.
\end{abstract}

Conclusions: Our study revealed both genetic and epigenetic mechanisms involved in duplicated gene silencing in the allotetraploid O. minuta.

Keywords: Comparative genomics, Wild rice, Allotetraploid, Gene silencing

\section{Background}

Rice is one of the most important crops for human consumption, as it feeds more than half of the world's population. To facilitate rice improvement, wild relative species in the genus Oryza have been employed as excellent genetic resources for rice breeding and genetic modification [1,2].

The genus Oryza, comprising two cultivated and approximately 22 wild species, is classified into 10 genome types, including six diploids (AA, BB, CC, EE, FF and GG) and four allotetraploids (BBCC, CCDD, HHJJ and KKLL) [3,4].

\footnotetext{
* Correspondence: mschen@genetics.ac.cn

${ }^{\dagger}$ Equal contributors

'State Key Laboratory of Plant Genomics, Institute of Genetics and Developmental Biology, Chinese Academy of Sciences, Beijing, China Full list of author information is available at the end of the article
}

To better exploit the superior wild rice genetic resources, a robust analysis of phylogeny among Oryza species was performed several years ago [4]. Subsequently, efforts have focused on deciphering the evolutionary relationship among diploid Oryza species [5-7].

However, within the genus Oryza, almost one-third of rice species are considered to be allotetraploids, representing a large part of species diversity present in this genus [4]. Elucidating the evolutionary history of allotetraploids in Oryza is highly important for obtaining a complete understanding of the evolution of Oryza. Unfortunately, the potential progenitors of only a few species with the BBCC genome type have been identified $[4,8]$. Of these species, O. minuta was selected as the representative species of BBCC in the Oryza Map Alignment

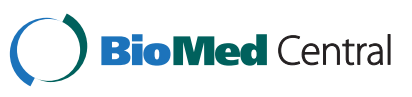


Project (OMAP) [2]. Comparative genomics resources are available, including a high quality bacterial artificial chromosome (BAC) library, BAC end sequences and a BAC-based physical map [9]. The genome donors of $O$. minuta were identified as diploid O. punctata (BB) and O. officinalis (CC), although some studies suggest that an extinct Asian $\mathrm{BB}$ genome carrier is the direct genome donor [10]. Several studies have deduced the molecular timing of BBCC formation, indicating that the formation of the allotetraploid occurred $\sim 0.3$ to 0.6 million years ago (Mya) $[8,10,11]$.

Polyploidy and the consequences of duplicated genomes have been extensively studied in some model species [12-17]. However, few studies have investigated microstructural variations using a comparative genomics approach [18-20]. The evolutionary fate of duplicated genes has also been well-studied, and expression analysis has often allowed gene silencing to be detected [21-25]. However, the genetic and epigenetic regulatory pathways of gene expression divergence are still largely unknown.

Shattering4 (Sh4), a major quantitative trait locus responsible for rice grain shattering, which encodes a transcription factor with an MYB3 DNA binding domain, plays an important role in the establishment of the abscission layer [26]. An amino acid substitution in the Sh4 coding region affects the normal development of abscission between the grain and the pedicel and further reduces grain shattering. Human selection of this single substitution promoted the domestication of rice from wild species. To deepen our understanding of the evolution of $O$. minuta, we conducted a comparative genomic analysis of a genomic segment surrounding the Sh4 locus among O. minuta, O. punctata and O. officinalis.

\section{Results}

Sequencing and annotation of the Sh4-orthologous regions We sequenced 10 BAC clones covering the Sh4-orthologous regions in O. punctata (BB), O. minuta (BBCC) and $O$. officinalis (CC) (Table 1). First, we refined the gene models in O. sativa ssp. japonica cv. Nipponbare (AA). After excluding eleven retrotransposon genes and nine hypothetical genes (Additional file 1: Table S1), the remaining 70 japonica reference genes were used to annotate genes in the other genomes using a multiple sequence comparative approach, as described in the Methods. A total of 207 manually revised gene models in four sets of genomes (BB, CC and $\mathrm{BBCC}$ ) were identified, including four putative pseudogenes (Additional file 1: Table S2). Comparative genomic analysis revealed that orthologous genes are well-conserved across all genomes (Figure 1).

Ten tandem duplicated gene clusters were identified within the Sh4 region, and copy number changes (CNCs) were found in four of these gene clusters $(16,29,39$ and 54). Among these genes, two tandem gene families (29 and 54; represented by purple and light blue bars in Figure 1, respectively) lost one copy in the polyploid (Additional file 1: Table S2). We investigated the evolutionary mechanism of the Gene 29 cluster, where three copies of Gene 29 were present in the AA and BBCC_CC genomes, four copies were present in the $\mathrm{BB}, \mathrm{BBCC} \mathrm{BB}$ and $\mathrm{CC}$ genomes and only one copy was present in the FF genome (O. brachyantha is the single representative of this genome type, which is the basal lineage in the genus Oryza). We found that multiple rounds of segmental duplication occurred in these regions to form this tandem gene cluster and they then evolved separately (Figure 2). Phylogenetic analysis indicated that Gene 29-4 is the most ancient copy and was stably maintained in the FF genome and selectively deleted in the AA genome (Additional file 1: Figure S1). Since we have no sequence information about Gene 54-2 in the $\mathrm{BB}$ and $\mathrm{CC}$ genome to ascertain whether this gene was deleted in BBCC_CC or duplicated in the other genomes, we searched for japonica Gene 54 by BLASTN against the O. brachyantha genome (FF) [11] and recently generated a draft sequence of $O$. puncata (unpublished data). We identified two tandem duplicates of Gene 54 and observed conserved gene organization in

Table 1 Sequenced BAC clones from the Sh4 region

\begin{tabular}{llllll}
\hline Species & Genome type & BAC clones & BAC length (bp) & Gaps & Total sequence length (bp) $^{*}$ \\
\hline O. punctata & BB & OP_Ba0062J15 & 161,916 & 2 & 367,482 \\
O. punctata & BB & OP_Ba0087M09 & 208,343 & 3 & - \\
O. officinalis & CC & OO_Ba0051G09 & 162,998 & Finished & 415,068 \\
O. officinalis & CC & OO_Ba0021120 & 256,074 & 3 & - \\
O. minuta (BB) & BBCC_BB & OM_Ba0107C17 & 107,440 & Finished & 403,493 \\
O. minuta (BB) & BBCC_BB & OM_Ba0002F21 & 137,910 & 3 & - \\
O. minuta (BB) & BBCC_BB & OM_Ba0232O12 & 184,883 & 1 & - \\
O. minuta (CC) & BBCC_CC & OM_Ba0113102 & 102,513 & Finished & 353,205 \\
O. minuta (CC) & BBCC_CC & OM_Ba0142K17 & 135,139 & 4 & - \\
O. minuta (CC) & BBCC_CC & OM_Ba0186E24 & 123,691 & 1 & - \\
\hline
\end{tabular}

*Total length of each genome after removing overlapping sequences. 


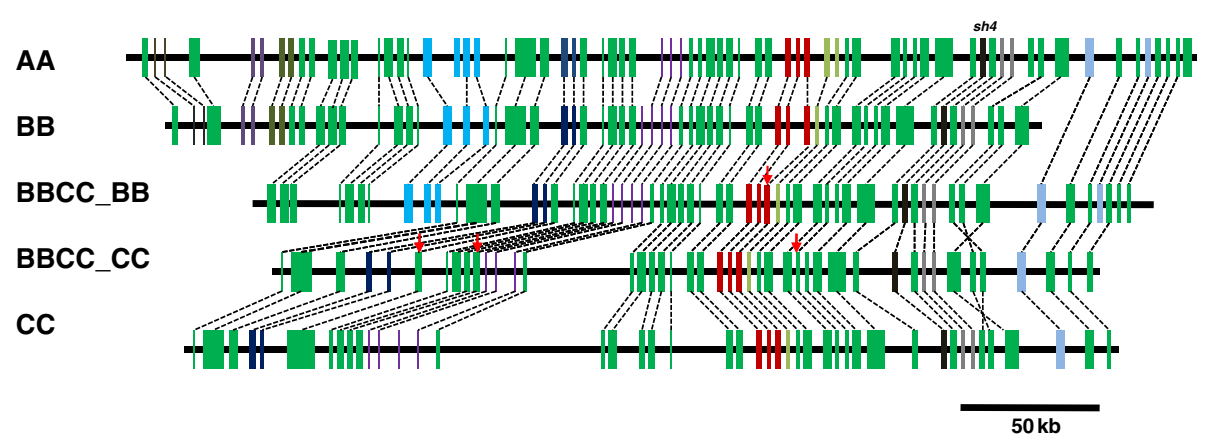

Figure 1 Gene collinearity across the genus Oryza. Orthologous genes in the Sh4 regions of AA, BB, BBCC and CC were aligned with each other. The genome structure is well-conserved between each subgenome of allotetraploid and its counterpart diploid. Ten tandemly duplicated gene clusters were found within this region and four putative pseudogenes were identified. The green bars represent single copy genes in this genomic region, the black bars represent the Sh4 gene, the other colored bars represent tandemly repeat genes and the red arrows indicate pseudogenes.

these two genomes, suggesting that this tandem duplication probably occurred before Oryza diversification. Phylogenetic analysis supports this notion: all 54-1 and 54-2 copies are separately clustered (Additional file 1: Figure S2). However, it cannot be confirmed whether one copy of Gene 54-2 was deleted from BC_C after polyploid formation or whether it was deleted from $\mathrm{CC}$ before polyploid formation, since sequence information for the CC genome is lacking. We designed 54-2-specific primers to amplify this copy in the CC genome, and no product could be detected, implying that this copy was probably deleted from the $\mathrm{CC}$ genome before polyploid formation (data not shown).

The details of repeat annotation are shown in Additional file 1: Table S3 and S4. We calculated and compared the TE contents for DNA type, RNA type and total (DNA and RNA) among O. minuta and two diploids. In BBCC_BB, the retrotransposon and total TE contents have been increasing compared with $\mathrm{BB}$, while the retrotransposon and total TE contents have been decreasing in BBCC_CC compared with the CC genome (Additional file 1: Figure S3). We compared the contents of LTRs and solo LTRs between the polyploid and diploid genomes. The number of solo LTRs was much greater than that of LTRs within all genomes, which indicates that the Oryza genomes are experiencing contraction (Table 2).

\section{Structural variation after allotetraploid formation}

Sequence comparisons revealed a $\sim 40 \mathrm{~kb}$ inversion in the CC subgenome in O. minuta (Additional File 1: Figure S4). We identified two identical MuLE elements from two sides of this inverted segment and examined whether recombination between these two elements caused the inversion. Sequence comparisons revealed that the two elements shared identical TSD and TIR sequences, indicating that homologous recombination has occurred between these MuLEs, which indeed caused the elements to exchange
TSD and TIR sequences, and thus the genomic sequence became inverted (Figure 3 ).

Transposition driven by Pack-MuLEs is another factor that contributes to genome non-collinearity. We identified four transposition events that occurred in the O. minuta genome after polyploid formation. Notably, one PackMuLE in the BB subgenome captured sequence fragments from several other gene loci and became integrated into a novel predicted ORF. We failed to determine whether this ORF is functional using RT-PCR (data not shown). More interestingly, at the same region of the CC subgenomes, we identified identical type, independently inserted Pack-MuLEs with completely different captured genomic sequences (Figure 4), which indicates that these genome regions may contain preferentially inserted sequence sites for this MuLE.

\section{Duplicated gene evolution in 0 . minuta}

To investigate duplicated gene evolution, we chose genes that were covered by BAC sequencing in all of the genomes, and therefore genes before 17 and after 53 were excluded from the following analysis (Additional file 1: Table S2). The Sh4 regions contain 41 sets of orthologous genes (from Gene 17 to 53). Among these genes, only one deletion of Gene 29-2 was observed (represented with purple bars in Figure 1) in BBCC_CC, and both copies of the other duplicated genes were maintained. We also identified four genes that were putatively pseudogenized in the polyploid genome, which formed after polyploidy (Additional file 1: Figure S5).

We estimated the molecular evolutionary rate for duplicated genes and examined whether these genes had different rates of evolution after polyploid formation (Additional file 1: Table S5). The results from the relative rate tests suggest that most genes do not exhibit obviously different rates between the polyploid and diploids. To determine which type of selection was acting upon the duplicated genes, we calculated the ratio of non-synonymous 


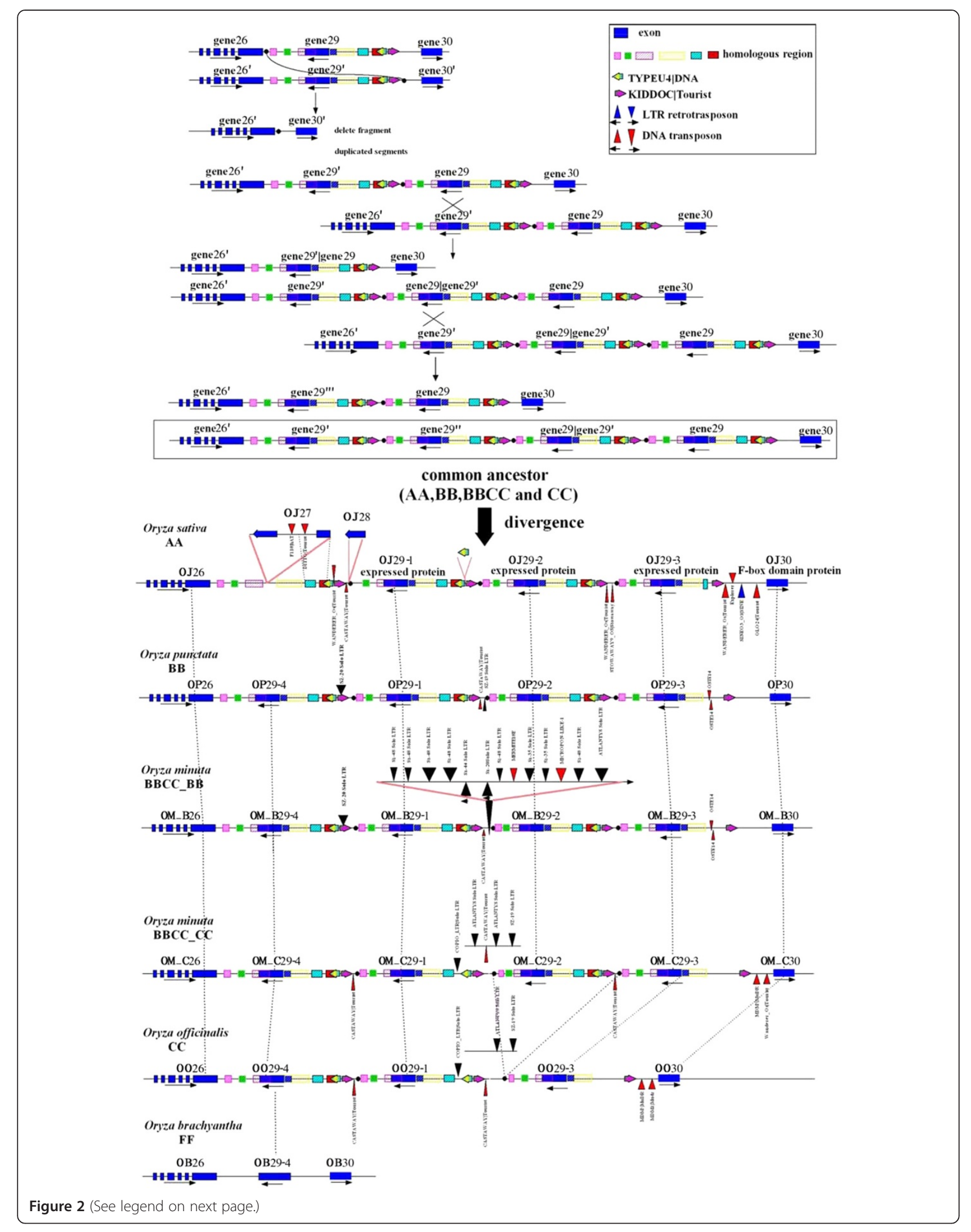


(See figure on previous page.)

Figure 2 Tandemly duplicated gene family formed through multiple rounds of segmental duplications. Genomic segments containing Gene 29 experienced three rounds of duplication, which led to the formation of the Gene 29 tandemly duplicated gene cluster. Subsequently, gene members were randomly deleted in some species after divergence among the genus Oryza during this evolutionary course. Gene 29 in 0. brachyantha, which is estimated to have separated from Oryza sativa 10 MYA, still maintains the ancient copy of Gene 29, and no amplifications or deletions were detected in this genome. OJ: O. sativa ssp. japonica, OP: O. punctata, OM_B: BB subgenome in O. minuta, OM_C: CC

subgenome in O. minuta and OO: O. officinalis.

(Ka) to synonymous (Ks) substitutions of protein coding sequences. Most duplicated genes were under purifying selection $(\mathrm{Ka} / \mathrm{Ks}<1)$, indicating that these genes are still strongly controlled after polyploidy (Additional file 1: Table S6). Similar patterns of Ks distribution between BB-BC_B and CC-BC_C also indicate that the evolutionary rates of duplicated genes were not obviously different (Additional file 1: Figure S6). We used duplicated genes to deduce the molecular timing of $O$. minuta divergence, and the results suggest that $O$. minuta was formed approximately 0.8-1.0 MYA (Additional file 1: Table S6), which is older than a previous estimate obtained by examining the Moc1 region ( 0.4 MYA) [11].

\section{Gene expression divergence of duplicated genes}

Since duplicated genes can exhibit significant variations in gene expression, we next examined the expression divergence of 34 sets of duplicated genes in O. minuta. We adopted the cDNA-SSCP assay to profile changes in gene expression, and considered only qualitative variations (i.e., expressed or silencing). The cDNA-SSCP results are shown in Figure 5, which shows that approximately 31\% $(9 / 29)$ of the genes have one silenced copy in BBCC. The complete results are shown in Additional file 1: Figure S7 and Table S7.

To investigate whether genetic or epigenetic mechanisms regulate these genes, we first examined the genomic sequences of silenced copies of these nine genes to determine whether or how the gene structures were destroyed. The silencing in only one gene (Gene 43 , whose CC subgenome copy was silenced) could be attributed to sequence variations (LTR insertion). In addition, we examined the expression patterns of two other genes (Gene 22 and Gene 26) that were identified as pseudogenes and found that both copies of the genes could be transcribed (coexpressed) (Additional file 1: Table S7). In summary, of the nine genes that exhibited expression silencing, only one was under genetic regulation. This result implies that other mechanisms, such as epigenetic regulation, may be involved in gene silencing.

DNA methylation, histone modification and other epigenetic modifications can affect gene expression $[27,28]$. Of these regulatory mechanisms, DNA methylation is one of the most common regulator of gene expression [29]. DNA methylation profiling of whole genomes has demonstrated that the methylation levels around the transcription start sites (TSS) of genes are quite low, indicating that there is a correlation between transcription and methylation $[30,31]$. Thus, we selected two sets of duplicated genes to determine whether the DNA methylation levels of regulatory sequences differ between normally expressed genes and silenced genes in the polyploid. For the first gene (Gene 17), we did not observed differential methylation in the regulatory regions between the two homoelogous genes. However, for the second gene (Gene 19), we detected differential methylation profiles between the duplicated genes. A cDNA-SSCP test indicated that Gene19 is normally expressed in the $\mathrm{CC}$ subgenome but silenced in the BB subgenome (Additional file 1: Figure S7). Therefore, we tested the methylation levels of approximately $2 \mathrm{~kb}$ upstream regions from the first exon, and detected heavy methylation near the TSS region of the BB copy of

Table 2 Summary of the ratio of LTRs and solo LTRs between the allotetraploid and diploids

\begin{tabular}{|c|c|c|c|c|c|c|c|}
\hline \multirow{2}{*}{$\begin{array}{l}\text { Pair-wise genome } \\
\text { comparison }\end{array}$} & \multirow[t]{2}{*}{ Genome specificity } & \multirow{2}{*}{$\begin{array}{l}\text { Intact } \\
\text { LTR-RT }\end{array}$} & \multirow{2}{*}{$\begin{array}{l}\text { Solo } \\
\text { LTR }\end{array}$} & \multirow{2}{*}{$\begin{array}{l}\text { Intact } \\
\text { solo LTR }\end{array}$} & \multicolumn{2}{|c|}{ MYA } & \multirow{2}{*}{$\begin{array}{l}\text { Intact LTR_RT: } \\
\text { Solo LTR (AII) }\end{array}$} \\
\hline & & & & & Range & Average & \\
\hline \multirow[t]{3}{*}{ BB VS BBCC_BB } & Only present in $\mathrm{BBCC} \_\mathrm{BB}$ & 2 & 16 & 1 & $0.781-4.035$ & 2.477 & $1: 15$ \\
\hline & Only present in BB & 1 & 8 & 0 & 0.889 & 0.89 & $1: 22$ \\
\hline & Both present & 0 & 14 & 2 & 0 & 0 & $0: 14$ \\
\hline \multirow[t]{3}{*}{ BBCC_CC VS CC } & Only present in BBCC_CC & 1 & 24 & 0 & 1.723 & 1.723 & $1: 60$ \\
\hline & Only present in CC & 3 & 24 & 2 & $0.254-5.215$ & 1.6821 & $1: 15$ \\
\hline & Both present & 1 & 36 & 4 & $0.553-1.723$ & 1.138 & $1: 36$ \\
\hline \multirow[t]{3}{*}{ BBCC_BB VS BBCC_CC } & Only present in BBCC_BB & 2 & 29 & 3 & $0.781-4.035$ & 2.477 & $1: 15$ \\
\hline & Only present in $\mathrm{BBCC}_{-} \mathrm{CC}$ & 1 & 59 & 4 & 1.723 & 1.723 & $1: 60$ \\
\hline & Both present & 0 & 1 & 0 & 0 & 0 & $0: 1$ \\
\hline
\end{tabular}




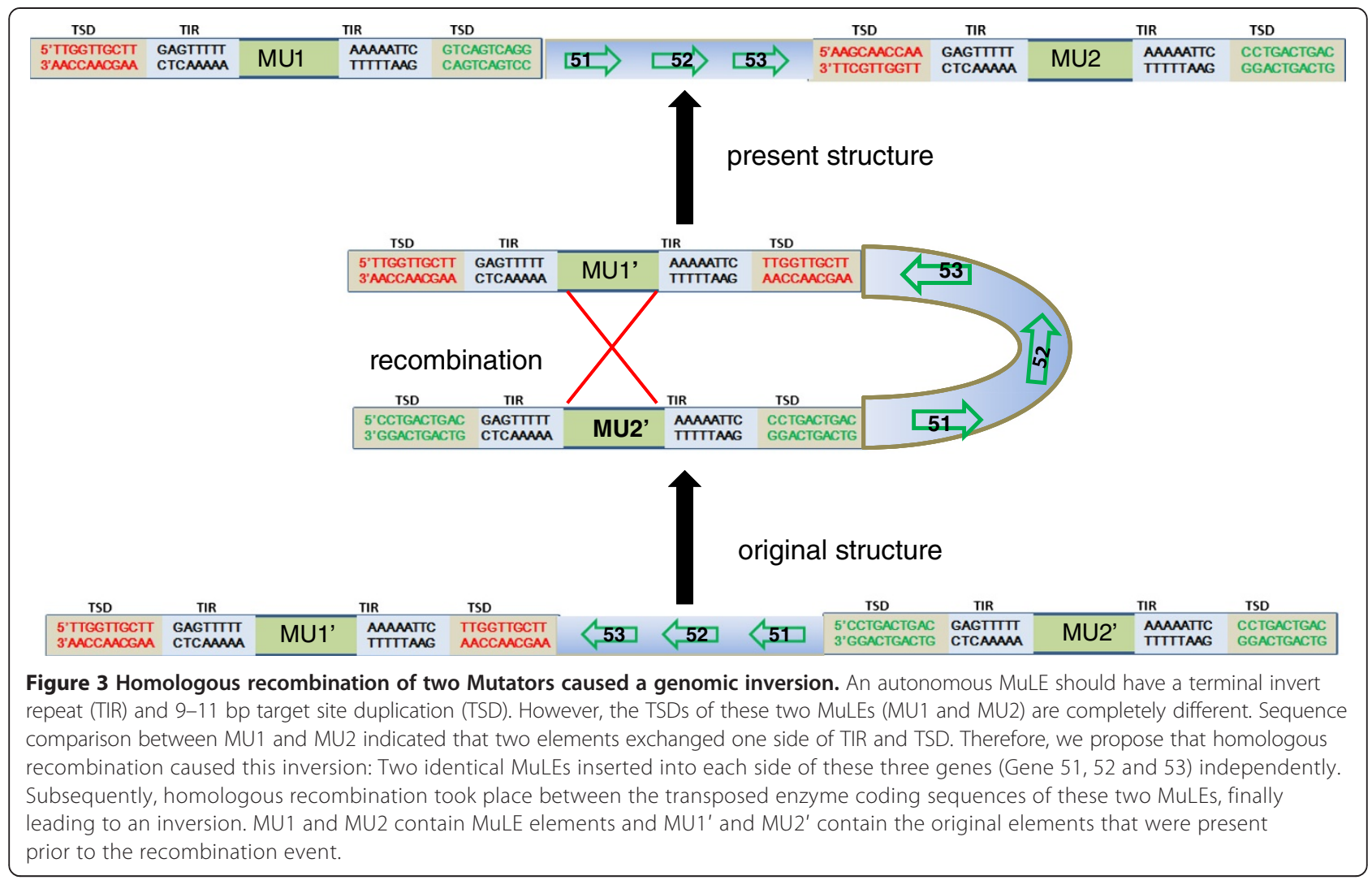

the gene but almost no methylation in the CC subgenome, suggesting that this regional, heavy DNA methylation probably caused the gene silencing (Figure 6). We also examined the orthologous regions in $\mathrm{BB}$ and $\mathrm{CC}$. No hypermethylation was found in either genome, suggesting that DNA methylation in this genomic region was not genetically inherited from the parental genomes, but it evolved after separation. We therefore examined whether the heavy methylation occurred spontaneously or whether there were other reasons for the phenomenon. When we compared the genomic sequences of this methylated region, we found that some of the sequences of BBCC_CC were completely absent from the other genomes. Annotation suggested that a $h A T$ element was specifically inserted into the CC subgenome. Therefore, this heavy DNA methylation was probably brought about by TE insertion, which is in accordance with the fact that methylation is a common approach used by genomes to defend themselves against TEs [29].

\section{Discussion}

Although several studies on synthetic and natural polyploids have provided evidence for rapid loss and gain of genomic segments (including genes) and extensive genomic reshuffling [14,16,25,32,33], sequence comparisons in the $S h 4$ genomic region, combined with the results from analysis of $A d h 1$ and $M o c 1$ [8,11], suggest that the natural
Oryza allotetraploid O. minuta is perhaps a relatively stable polyploid. Such stabilization is supported by the presence of conserved genes, intergenic regions and even shared TEs between polyploid and diploid genomes. However, confirming this notion would require additional investigations of other larger segments or even whole genomes.

The regulation of duplicated gene expression in polyploids has been well-studied in several model species, but few of these studies have correlated expression divergence with sequence variations $[10,23,34-36]$. In this study, we found that two pairs of duplicated genes annotated as pseudogenes (22 and 26) could be coexpressed in a cDNA-SSCP assay. Analysis of cDNA sequences has indicated that transcripts from pseudogenes are non-functional. Therefore, coexpression of homoelogous genes revealed by microarray analysis (or other methods) does not guarantee that both gene copies are treated equally by the genome, as mRNA sequence variations cannot be detected using these approaches. Notably, the biological significance of pseudogenes has recently been examined, especially pseudogenes that can be transcribed $[37,38]$. These findings suggest that pseudogenes can probably evolve from being buried in huge genomes to becoming new, functional elements, implying that pseudogenization can lead to neofunctionalization.

Gene expression divergence was found to be more prevalent in $\mathrm{BBCC}$ than was previously predicted in other species [21]. Both genetic and epigenetic regulatory 


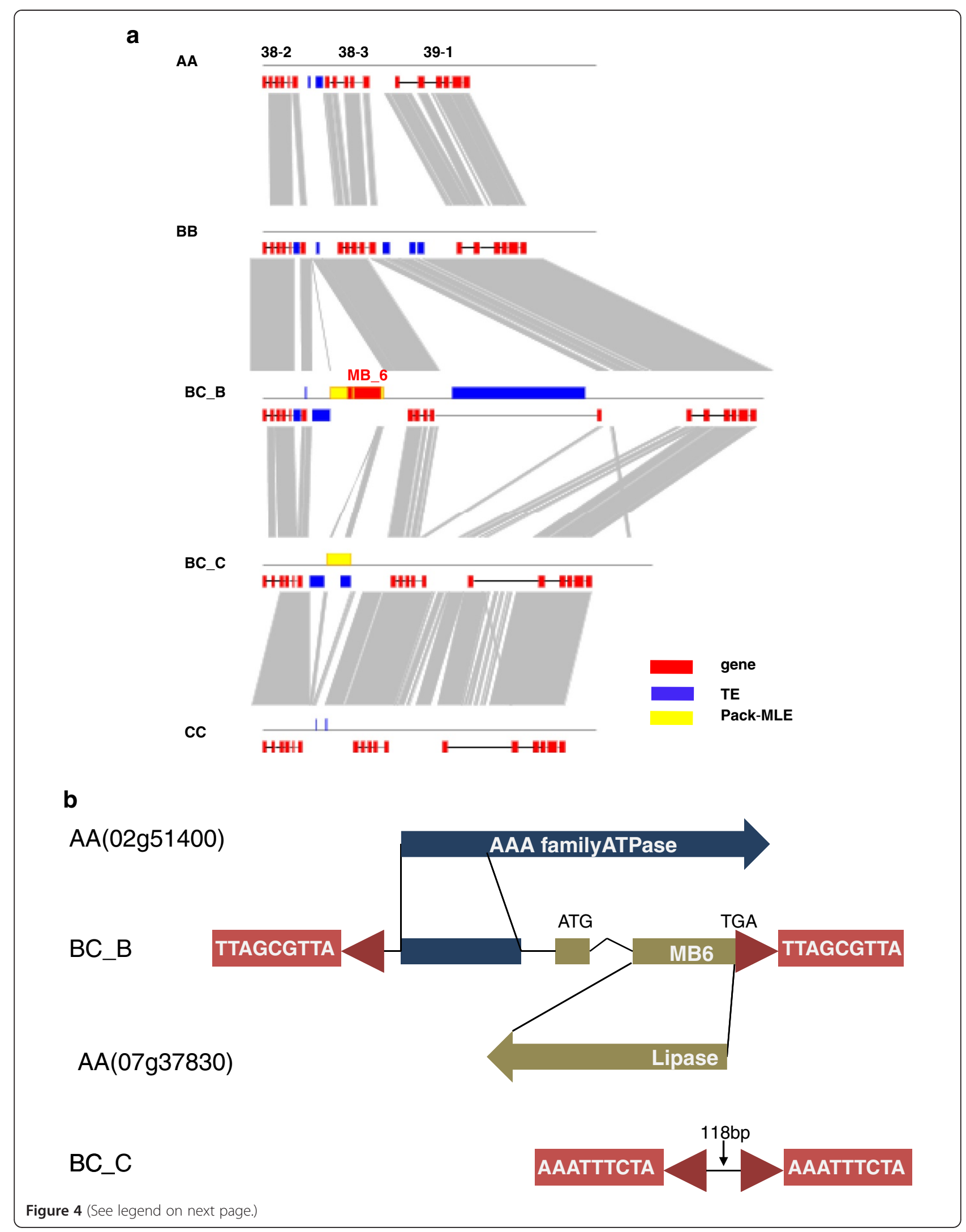


(See figure on previous page.)

Figure 4 Pack-MuLEs movements in allotetraploid Oryza minuta and its diploid ancestors. Through comparative genomic analysis, we identified one Pack-MuLE specifically inserted into the BBCC_BB subgenome. FGENESH predicted one specific gene model within this MuLE element, named MB6 (a). We aligned the captured sequence of this MuLE with the japonica reference genome and found that this MuLE probably captured sequences from multiple loci. This region ( 1,100 bp) has high identity with $07 \mathrm{~g} 37840$ ( $94 \%$ identity), which produced a novel ORF by integration with some unknown sequences (b). At the same genomic region of the CC subgenome in O. minuta, we identified the same type of MuLE with different foreign sequences (118 bp in length and lacking an exact homologous sequence in the japonica genome) (a). These two MuLEs share a highly identical 3 ' sequence and a relatively divergent $5^{\prime}$ sequence (probably caused by other TE insertions). The presence of completely different TSD sequences suggests that these insertion events occurred independently (b).

mechanisms were found to control duplicated genes in O. minuta. Deletion is a robust mechanism for controlling duplicated genes, but it is not a universal approach, as observed within the $S h 4$ region, and it often occurs with multiple copy genes. Sequence analysis to detect gene functional deficiency caused by structural variation has revealed that only one gene is regulated genetically, implying that more genes may be regulated by epigenetic mechanisms. The example of TE-driven methylation silencing illustrates a potential epigenetic gene silencing pathway, which occurs as follows: after polyploidy, the presence of a duplicated genome decreases the stress upon TEs, which are then reactivated and become randomly inserted into the genome. Once integrated into sensitive genome regions (such as regulatory or translated sequences) and simultaneously supervised by the epigenetic network (e.g., DNA methylation), the function (or expression) of nearby genes may be affected to various extents

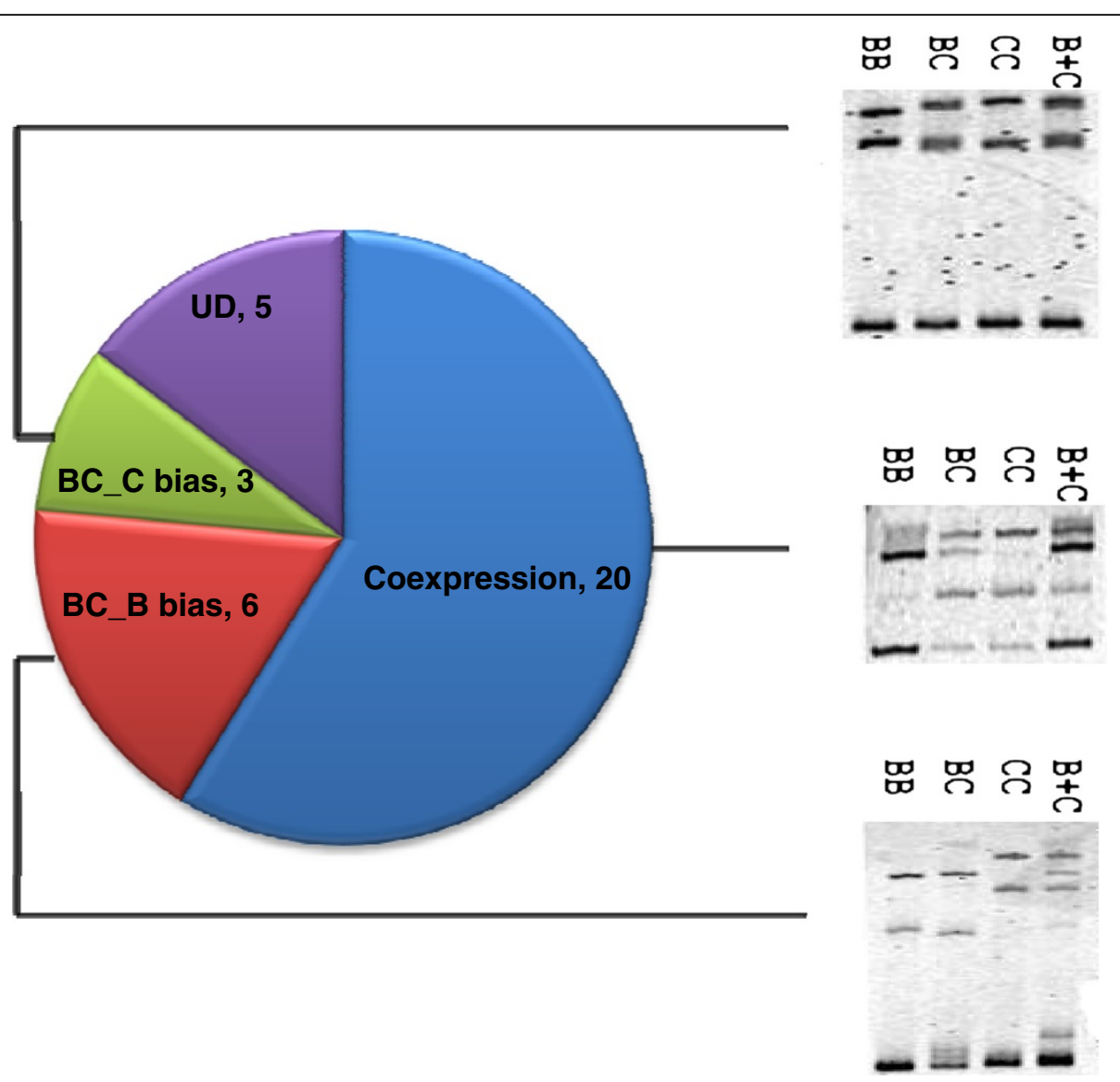

Figure 5 The expression patterns of homoeologous gene pairs revealed by CDNA-SSCP analysis. We examined a total of 34 duplicated genes to investigate the variation in gene expression patterns between the genes in each pair. The CDNA-SSCP analysis of three different types of duplicated genes, combined with PAGE, was used to reveal divergence in gene expression patterns among genes (Gene 19 represented BBCC_CC bias, Gene 43 represented BBCC_BB bias and Gene 22 represented coexpressed). Coexpressed: both copies are expressed normally; BBCC_BB bias: copy from the BB subgenome is expressed, but the one from the CC subgenome is not expressed; BBCC_CC bias: copy from the CC subgenome is expressed, but the one from the BB subgenome is not expressed. 


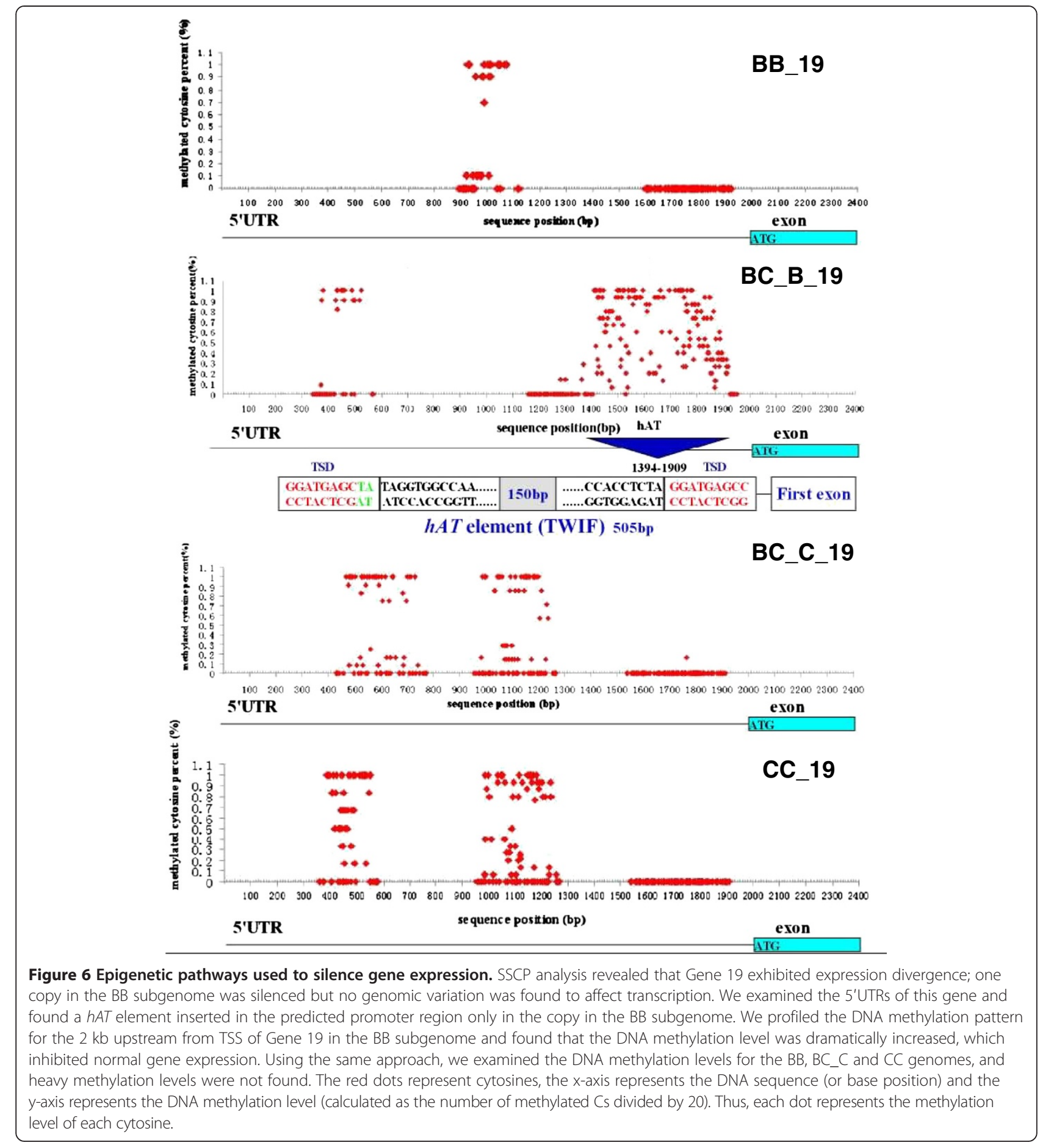

and may even become completely inactivated (Figure 7). This epigenetic regulation should be more prevalent in polyploids than diploids, since it is much more difficult for this kind of high-impact insertions to escape from selection in diploids than in polyploids.

Although genome-wide experimental data are required to prove that the epigenetic pathway is the universal mechanism for gene silencing, it is currently more difficult to test epigenetic markers than genetic sequences, especially when dealing with polyploids. Nonetheless, we still propose that DNA methylation-controlled gene silencing is a prevalent mechanism, based on several observations. First, TEs have been reported to regulate gene expression for numerous genes $[39,40]$. For instance, a SINE element inserted upstream of the FWA coding sequence caused this gene to be epigenetically silenced 


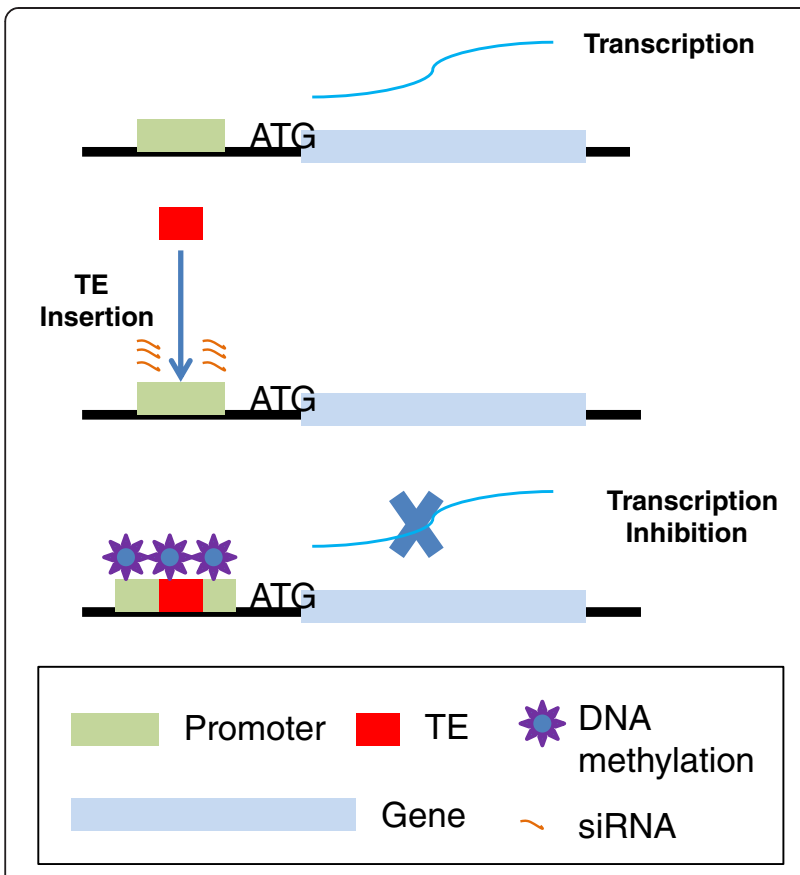

Figure 7 Model showing how TE-driven DNA methylation leads to silencing of duplicated genes.

in vegetative tissues of Arabidopsis [41]. Second, numerous TEs are likely to be inserted adjacent to genes in the BBCC genome. TEs can affect gene expression, but the effects of TEs decrease with increasing distance between the TEs and genes [42]. We investigated the distribution of several types of DNA-type TEs in the japonica genome. These TEs have the greatest potential to regulate nearby genes due to their preferential insertion near or within genes. Three types of TEs, including hAT, Stowaway and Tourist, are all present in thousands of copies $(6,728,49,810$ and 40,092, respectively), and the mean distance of these TEs to genes is within $2 \mathrm{~kb}$ (data not shown). Therefore, we postulate that a considerable number of potential epigenetic triggers have also been buried within the BBCC genome. More importantly, according to previous reports and the results of the current study, TE insertion is not sufficient to initiate the silencing pathway; siRNA or DNA methylation is essential for initiating this program. For example, the same $h A T$ elements were found in regulatory regions of the FLC gene in two Arabidopsis ecotypes, Landsberg erecta (Ler) and Columbia (Col). However, the roles of these two $h A T$ elements in regulating FLC gene expression are quite different, as $\sim 24-$ nt siRNA was found at higher levels in Ler than in Col; this siRNA can mediate DNA methylation and gene silencing in the Ler ecotype [43]. Recently, the global effects of TEs on gene expression were investigated in Arabidopsis and its close relative. Genome-wide analysis indicated that TEs can affect the expression of nearby genes, especially when the TEs are epigenetically modified (methylated or siRNA targeted) [42,44]. We also calculated the methylation rates of the above three types of TEs in the japonica genome and found that methylated copies account for over $80 \%$ of these TEs, providing further support for the potential role of silenced TEs in gene regulation. Here, we used the japonica genome to represent $O$. minuta. Future studies should focus on comprehensive analysis of the $O$. minuta genome to help elucidate the epigenetic regulatory pathway on a genomewide scale.

\section{Conclusions}

By integrating comparative genomic tools, gene expression and epigenetic analysis, our study comprehensively demonstrates how duplicated genomes and genes evolve within the Sh4 region in O. minuta. We found that duplicated genes are under both genetic and epigenetic regulation, and DNA methylation is proposed as a potentially important regulatory mechanism for gene silencing.

\section{Methods}

\section{Plant materials and BAC library}

Seeds and seedlings of Oryza minuta (Accession No.101141), O. punctata (Accession No.105690) and O. officinalis (Accession No.100896) were obtained from the International Rice Resource Institute (IRRI, Philippines). High-density BAC library filters and BAC clones for three Oryza species were purchased from the Arizona Genomics Institute (USA).

\section{$B A C$ identification and sequencing}

BAC clones covering the orthologous regions of the japonica Sh4 genome segment were identified by screening Oryza genomic BAC libraries following the method described by Lu et al. [11]. Initial selections were conducted using two unique probes (designed with two japonica gene models, LOC_Os04g57350 and LOC_Os04g57600, which are located upstream and downstream of the Shattering 4 [LOC_Os04g57530] gene locus, respectively), to hybridize to high-density filters containing three Oryza genomic BAC libraries. Combined with physical map positions in Finger Printed Contigs (FPC), a total of 98 positive BAC clones were identified. All screened BAC clones were digested with HindIII, size-selected by electrophoresis and transferred onto nylon filters for Southern blot analysis. For diploid genomes, eight additional probes were used to identify the BAC clones, which maximized the orthologous region coverage and minimized the gaps between consecutive BAC clones. To distinguish between the subgenomes of $O$. minuta, the digested map of each BAC was compared with that of O. punctata and $O$. officinalis. The tetrapolyploid BAC clones were divided into two groups (each from one parental genome) based on their HindIII digestion patterns. Ten BAC clones of three Oryza species were sequenced with an ABI 3730 
automated sequencer (Table 1). Specifically, Sanger reads were assembled from each BAC into contigs, and BAC sequences were then merged from the same genome by identifying overlapping sequences (Table 1). Orthologous genome regions in $O$. brachyantha (FF) were recently generated [45].

\section{Genome annotation}

A comparative gene annotation approach was taken to identify gene models in the $\mathrm{BB}, \mathrm{CC}$ and $\mathrm{BBCC}$ genomes. Before using the japonica Sh4 genome annotation as a reference gene model, transposon-related gene models and hypothetical genes without cDNA, ESTs and homologous proteins were excluded. Gene structures were confirmed using full-length japonica cDNA. To annotate nonjaponica genomes, four wild rice genomes were initially repeat-masked and predicted using FGENESH (http:// linux1.softberry.com). All predicted genes were aligned with japonica cDNA and proteins from rice, Sorghum, Brachypodium and Maize. Gene models without cDNA or protein supports were excluded. All gene models were manually refined based on the AA-BB-BBCC-CC multiple alignment framework. Conserved gene structures were modified based on japonica gene models. RT-PCR was used to detect gene structure if great variation existed between japonica genes and non-japonica genes. Multiple sequence comparisons were performed with CLUSTALW. Structural variation was detected using Artemis Comparison Tool (ACT) [46].

To identify transposon elements, RepeatMasker was initially used to annotate the TEs and other repeat sequences, followed by manual analysis (http://www.repeatmasker.org/). LTR-type transposons were also predicted with LTR_STRUC [47], LTR_Finder [48] and LTRharvest [49] to complement the results from RepeatMasker. Intact structures and other TE signatures such as target site duplication (TSD), terminal inverted repeats (TIR), polypurine tracts (PPT), primer binding sites (PBS) and long terminal repeats (LTR) were manually identified using Dotter software [50] and ACT. The insertion time of each LTR was estimated using the baseml program in PAML at a mutation rate of $1.3 \times 10^{-8}$ per site per year [51].

Genomic structural variations, such as inversions, were also detected by ACT, followed by thorough analysis of their boundary sequences. Pack-MuLE elements were annotated manually, and to determine the captured genome sequence in the wild rice genome, the homologous sequences were searched against the japonica reference genome.

\section{Molecular evolution analysis of duplicated genes}

To determine the type of selection acting upon duplicated genes, $K a$ and $K s$ values were calculated for duplicated genes in O. minuta using the baseml program with the pairwise model in PAML version 4.6 [52]. Alignments for coding sequences of duplicated genes were conducted with CLUSTALW [53]. Divergence times of duplicated genes were calculated with a synonymous substitution rate of $6.5 \times 10^{-9}$ substitutions per site per year [54]. Relative evolutionary rates of duplicated genes were estimated using the Tajima relative rate test implemented in MEGA v5.2 [55].

\section{CDNA-SSCP analysis}

Total RNA samples were isolated from six different tissues of three Oryza species, including mature leaves, mature roots, young leaves, young roots, flowers and mixed panicles. Reverse transcription was performed on mixed total RNA from all tissues, and cDNA products were used to amplify orthologous genes. SSCP analysis was then conducted with the Bio-RAD Dcode ${ }^{\mathrm{Tm}}$ system following the standard protocol (Bio-RAD, USA). The SSCP results revealed the gene expression patterns of duplicated genes in O. minuta, including both coexpressed and gene silencing. All primer sequences are listed in Additional file 2.

\section{Methylation-specific PCR analysis}

DNA was extracted from mature O. punctata, O. officinalis and $O$. minuta leaves, digested with restriction enzymes and treated with sodium bisulfite to convert the unmethylated cytosine residues to uracil. A set of primers was designed to amplify the genomic region of each gene from the end of the first exon to $\sim 2 \mathrm{~kb}$ upstream of the gene; the size of each PCR product was approximately 150-300 bp. Primer sequences are listed in Additional file 2. The products were recovered, cloned and sequenced for DNA methylation analysis.

\section{Availability of supporting data}

Sh4 genome sequences for wild rices can be downloaded from NCBI at http://www.ncbi.nlm.nih.gov/nuccore/ HQ827834 (BB), http://www.ncbi.nlm.nih.gov/nuccore/HQ82 7835 (BC_B), http://www.ncbi.nlm.nih.gov/nuccore/HQ827836 (BC_C) and http://www.ncbi.nlm.nih.gov/nuccore/HQ827837 (CC). O. sativa ssp. japonica sequences can be downloaded from http://rice.plantbiology.msu.edu/. FF genome can be downloaded from http://www.gramene.org/Oryza_brachyantha/Info/Index.

\section{Additional files}

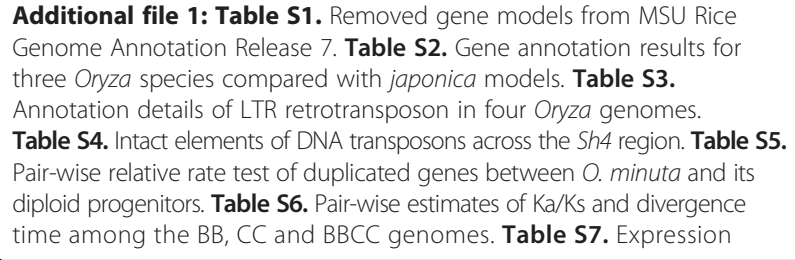

Additional file 1: Table S1. Removed gene models from MSU Rice Genome Annotation Release 7. Table S2. Gene annotation results for three Oryza species compared with japonica models. Table S3. Annotation details of LTR retrotransposon in four Oryza genomes. Table S4. Intact elements of DNA transposons across the Sh4 region. Table S5. Pair-wise relative rate test of duplicated genes between 0 . minuta and its diploid progenitors. Table S6. Pair-wise estimates of Ka/Ks and divergence time among the BB, CC and BBCC genomes. Table S7. Expression 
divergence of duplicated genes in allotetraploid O. minuta. Figure S1. Phylogenetic analysis of Gene 29 clusters. Figure S2. Evolutionary analysis of Gene 54 clusters. Figure S3. TE contents in five Oryza species. Figure S4. Large inversion detected in the CC subgenome of $\mathbf{O}$. minuta. Figure $\mathbf{S 5 .}$ Pseudogene annotation. Figure S6. Distributions of Ks density for homoeologous genes between two subgenomes in O. minuta and corresponding diploid genomes. Figure $\mathbf{S 7}$. Results of CDNA-SSCP analysis with 34 pairs of duplicated genes.

Additional file 2: Primer information in this study.

\section{Competing interests}

The authors declare that they have no competing interests.

\section{Authors' contributions}

MSC designed and managed the project. YS and JFS prepared the materials and generated sequence data and performed the CDNA-SSCP analysis. YS and $B L$ analyzed the data and performed epigenetic experiments. $B L$ and MSC wrote the manuscript. All authors read and approved the final manuscript.

\section{Acknowledgements}

This work was supported by the National Natural Science Foundation of China (grants \# 31171231 and 30770143) to MSC.

\section{Author details}

${ }^{1}$ State Key Laboratory of Plant Genomics, Institute of Genetics and Developmental Biology, Chinese Academy of Sciences, Beijing, China. ${ }^{2}$ Current address: Institute of Crop Science, Chinese Academy of Agricultural Sciences, Beijing, China.

Received: 25 August 2013 Accepted: 30 December 2013 Published: 6 January 2014

\section{References}

1. Vaughan DA, Morishima H, Kadowaki K: Diversity in the Oryza genus. Curr Opin Plant Biol 2003, 6(2):139-146.

2. Wing RA, Ammiraju JS, Luo M, Kim H, Yu Y, Kudrna D, Goicoechea JL, Wang W, Nelson W, Rao K: The Oryza map alignment project: the golden path to unlocking the genetic potential of wild rice species. Plant Mol Biol 2005, 59(1):53-62.

3. Aggarwal R, Brar D, Nandi S, Huang N, Khush G: Phylogenetic relationships among Oryza species revealed by AFLP markers. Theor Appl Genet 1999, 98(8):1320-1328

4. Ge S, Sang T, Lu B-R, Hong D-Y: Phylogeny of rice genomes with emphasis on origins of allotetraploid species. Proc Natl Acad Sci 1999 96(25):14400-14405.

5. Zhu Q, Ge S: Phylogenetic relationships among A-genome species of the genus Oryza revealed by intron sequences of four nuclear genes. New Phytol 2005, 167(1):249-265.

6. Zhu Q, Zheng X, Luo J, Gaut BS, Ge S: Multilocus analysis of nucleotide variation of Oryza sativa and its wild relatives: severe bottleneck during domestication of rice. Mol Biol Evol 2007, 24(3):875-888.

7. Zou XH, Zhang FM, Zhang JG, Zang LL, Tang L, Wang J, Sang T, Ge S: Analysis of 142 genes resolves the rapid diversification of the rice genus. Genome Biol 2008, 9(3):R49.

8. Ammiraju JSS, Fan C, Yu Y, Song X, Cranston KA, Pontaroli AC, Lu F, Sanyal A, Jiang N, Rambo T: Spatio-temporal patterns of genome evolution in allotetraploid species of the genus Oryza. Plant J 2010, 63(3):430-442.

9. Kim H, Hurwitz B, Yu Y, Collura K, Gill N, SanMiguel P, Mullikin JC, Maher C, Nelson W, Wissotski M: Construction, alignment and analysis of twelve framework physical maps that represent the ten genome types of the genus Oryza. Genome Biol 2008, 9(2):R45.

10. Wang B, Ding Z, Liu W, Pan J, Li C, Ge S, Zhang D: Polyploid evolution in Oryza officinalis complex of the genus Oryza. BMC Evol Biol 2009, 9(1):250.

11. Lu F, Ammiraju JS, Sanyal A, Zhang S, Song R, Chen J, Li G, Sui Y, Song X, Cheng Z: Comparative sequence analysis of MONOCULM1-orthologous regions in 14 Oryza genomes. Proc Natl Acad Sci 2009, 106(6):2071-2076.
12. Feldman M, Liu B, Segal G, Abbo S, Levy AA, Vega JM: Rapid elimination of low-copy DNA sequences in polyploid wheat: a possible mechanism for differentiation of homoeologous chromosomes. Genetics 1997, 147(3):1381-1387.

13. Soltis DE, Soltis PS: The dynamic nature of polyploid genomes. Proc Natl Acad Sci 1995, 92(18):8089-8091.

14. Song K, Lu P, Tang K, Osborn TC: Rapid genome change in synthetic polyploids of Brassica and its implications for polyploid evolution. Proc Natl Acad Sci 1995, 92(17):7719-7723.

15. Xiong Z, Gaeta RT, Pires JC: Homoeologous shuffling and chromosome compensation maintain genome balance in resynthesized allopolyploid Brassica napus. Proc Natl Acad Sci 2011, 108(19):7908-7913.

16. Chester M, Gallagher JP, Symonds W, Da Silva AVC, Mavrodiev EV, Leitch AR, Soltis PS, Soltis DE: Extensive chromosomal variation in a recently formed natural allopolyploid species, Tragopogon miscellus (Asteraceae). Proc Natl Acad Sci 2012, 109(4):1176-1181.

17. Gaeta RT, Pires JC, Iniguez-Luy F, Leon E, Osborn TC: Genomic changes in resynthesized Brassica napus and their effect on gene expression and phenotype. Plant Cell Online 2007, 19(11):3403-3417.

18. Comai L, Tyagi AP, Winter K, Holmes-Davis R, Reynolds SH, Stevens Y, Byers B: Phenotypic instability and rapid gene silencing in newly formed Arabidopsis allotetraploids. Plant Cell Online 2000, 12(9):1551-1568.

19. Fiebig A, Kimport R, Preuss D: Comparisons of pollen coat genes across Brassicaceae species reveal rapid evolution by repeat expansion and diversification. Proc Natl Acad Sci 2004, 101(9):3286-3291.

20. Grover CE, Kim HR, Wing RA, Paterson AH, Wendel JF: Microcolinearity and genome evolution in the AdhA region of diploid and polyploid cotton (Gossypium). Plant J 2007, 50(6):995-1006.

21. Adams KL, Cronn R, Percifield R, Wendel JF: Genes duplicated by polyploidy show unequal contributions to the transcriptome and organ-specific reciprocal silencing. Proc Natl Acad Sci 2003, 100(8):4649-4654

22. Adams KL, Wendel JF: Novel patterns of gene expression in polyploid plants. Trends Genet 2005, 21(10):539-543.

23. Pumphrey M, Bai J, Laudencia-Chingcuanco D, Anderson O, Gill BS: Nonadditive expression of homoeologous genes is established upon polyploidization in hexaploid wheat. Genetics 2009, 181(3):1147-1157.

24. Chen ZJ: Genetic and epigenetic mechanisms for gene expression and phenotypic variation in plant polyploids. Annu Rev Plant Biol 2007, 58:377-406.

25. Kashkush K, Feldman M, Levy AA: Gene loss, silencing and activation in a newly synthesized wheat allotetraploid. Genetics 2002, 160(4):1651-1659.

26. Li C, Zhou A, Sang T: Rice domestication by reducing shattering. Science 2006, 311(5769):1936-1939.

27. Henderson IR, Jacobsen SE: Epigenetic inheritance in plants. Nature 2007, 447(7143):418-424

28. Jaenisch R, Bird A: Epigenetic regulation of gene expression: how the genome integrates intrinsic and environmental signals. Nat Genet 2003, 33:245-254.

29. Chan SW-L, Henderson IR, Jacobsen SE: Gardening the genome: DNA methylation in Arabidopsis thaliana. Nat Rev Genet 2005, 6(5):351-360.

30. Cokus SJ, Feng S, Zhang X, Chen Z, Merriman B, Haudenschild CD, Pradhan S, Nelson SF, Pellegrini M, Jacobsen SE: Shotgun bisulphite sequencing of the Arabidopsis genome reveals DNA methylation patterning. Nature 2008, 452(7184):215-219.

31. Zemach A, McDaniel IE, Silva P, Zilberman D: Genome-wide evolutionary analysis of eukaryotic DNA methylation. Science 2010, 328(5980):916-919.

32. Liu B, Vega J, Feldman M: Rapid genomic changes in newly synthesized amphiploids of Triticum and Aegilops. II. Changes in low-copy coding DNA sequences. Genome 1998, 41(4):535-542.

33. Liu B, Vega J, Segal G, Abbo S, Rodova M, Feldman M: Rapid genomic changes in newly synthesized amphiploids of Triticum and Aegilops. I. Changes in low-copy noncoding DNA sequences. Genome 1998, 41(2):272-277.

34. Flagel L, Udall J, Nettleton D, Wendel J: Duplicate gene expression in allopolyploid Gossypium reveals two temporally distinct phases of expression evolution. BMC Biol 2008, 6(1):16.

35. Hovav R, Udall JA, Chaudhary B, Rapp R, Flagel L, Wendel JF: Partitioned expression of duplicated genes during development and evolution of a single cell in a polyploid plant. Proc Natl Acad Sci 2008, 105(16):6191-6195.

36. Kim E-D, Chen ZJ: Unstable transcripts in arabidopsis allotetraploids are associated with nonadditive gene expression in response to abiotic and biotic stresses. PLoS One 2011, 6(8):e24251. 
37. Pei B, Sisu C, Frankish A, Howald C, Habegger L, Mu XJ, Harte R, Balasubramanian S, Tanzer A, Diekhans M: The GENCODE pseudogene resource. Genome Biol 2012, 13(9):R51

38. Kalyana-Sundaram S, Kumar-Sinha C, Shankar S, Robinson DR, Wu Y-M, Cao X, Asangani IA, Kothari V, Prensner JR, Lonigro RJ: Expressed pseudogenes in the transcriptional landscape of human cancers. Cell 2012. 149(7):1622-1634.

39. Slotkin RK, Martienssen R: Transposable elements and the epigenetic regulation of the genome. Nat Rev Genet 2007, 8(4):272-285.

40. Lisch D: How important are transposons for plant evolution? Nat Rev Genet 2013, 14(1):49-61.

41. Kinoshita Y, Saze H, Kinoshita T, Miura A, Soppe WJ, Koornneef M, Kakutani T: Control of FWA gene silencing in Arabidopsis thaliana by SINE-related direct repeats. Plant J 2006, 49(1):38-45.

42. Hollister JD, Gaut BS: Epigenetic silencing of transposable elements: a trade-off between reduced transposition and deleterious effects on neighboring gene expression. Genome Res 2009, 19(8):1419-1428.

43. Zhai J, Liu J, Liu B, Li P, Meyers BC, Chen X, Cao X: Small RNA-directed epigenetic natural variation in Arabidopsis thaliana. PLoS Genet 2008, 4(4):e1000056.

44. Hollister JD, Smith LM, Guo Y-L, Ott F, Weigel D, Gaut BS: Transposable elements and small RNAs contribute to gene expression divergence between Arabidopsis thaliana and Arabidopsis lyrata. Proc Natl Acad SCi 2011, 108(6):2322-2327.

45. Chen J, Huang Q, Gao D, Wang J, Lang Y, Liu T, Li B, Bai Z, Luis Goicoechea J, Liang $C$, et al: Whole-genome sequencing of Oryza brachyantha reveals mechanisms underlying Oryza genome evolution. Nat Commun 2013, 4:1595.

46. Carver TJ, Rutherford KM, Berriman M, Rajandream MA, Barrell BG, Parkhill J: ACT: the Artemis comparison tool. Bioinformatics 2005, 21(16):3422-3423.

47. McCarthy EM, MCDonald JF: LTR_STRUC: a novel search and identification program for LTR retrotransposons. Bioinformatics 2003, 19(3):362-367.

48. Xu Z, Wang H: LTR_FINDER: an efficient tool for the prediction of full-length LTR retrotransposons. Nucleic Acids Res 2007, 35(suppl 2):W265-W268.

49. Ellinghaus D, Kurtz S, Willhoeft U: LTRharvest, an efficient and flexible software for de novo detection of LTR retrotransposons. BMC Bioinforma 2008, 9(1):18.

50. Sonnhammer ELL, Durbin R: A dot-matrix program with dynamic threshold control suited for genomic DNA and protein sequence analysis. Gene 1995, 167(1):GC1-GC10.

51. Ma J, Bennetzen JL: Rapid recent growth and divergence of rice nuclear genomes. Proc Natl Acad Sci U S A 2004, 101(34):12404-12410.

52. Yang Z: PAML 4: phylogenetic analysis by maximum likelihood. Mol Biol Evol 2007, 24(8):1586-1591.

53. Larkin M, Blackshields G, Brown N, Chenna R, McGettigan P, McWilliam $H$ Valentin F, Wallace I, Wilm A, Lopez R: Clustal W and Clustal X version 2.0. Bioinformatics 2007, 23(21):2947-2948.

54. Gaut BS, Morton BR, McCaig BC, Clegg MT: Substitution rate comparisons between grasses and palms: synonymous rate differences at the nuclear gene Adh parallel rate differences at the plastid gene rbcL. Proc Natl Acad Sci 1996, 93(19):10274-10279.

55. Tamura K, Peterson D, Peterson N, Stecher G, Nei M, Kumar S: MEGA5: molecular evolutionary genetics analysis using maximum likelihood, evolutionary distance, and maximum parsimony methods. Mol Biol Evol 2011, 28(10):2731-2739.

doi:10.1186/1471-2164-15-11

Cite this article as: Sui et al:: Genomic, regulatory and epigenetic mechanisms underlying duplicated gene evolution in the natural allotetraploid Oryza minuta. BMC Genomics 2014 15:11.

\section{Submit your next manuscript to BioMed Central and take full advantage of:}

- Convenient online submission

- Thorough peer review

- No space constraints or color figure charges

- Immediate publication on acceptance

- Inclusion in PubMed, CAS, Scopus and Google Scholar

- Research which is freely available for redistribution

Submit your manuscript at www.biomedcentral.com/submit
Ciomed Central 